\title{
Single dose cefoxitin in treating uncomplicated gonorrhoea caused by penicillinase producing Neisseria gonorrhoeae (PPNG) and non-PPNG strains
}

\author{
K B LIM,* T THIRUMOORTHY,* C T LEE,* S N THAM,* E H SNG, $†$ AND T TAN* \\ From the *Middle Road Hospital, and the †Department of Pathology, Singapore General Hospital, \\ Singapore
}

SUMMARY A total of 136 patients with uncomplicated gonorrhoea were treated with intramuscular cefoxitin $2 \mathrm{~g}$ (25 patients) or $1 \mathrm{~g}$ (111 patients) and oral probenecid $1 \mathrm{~g}$. Cefoxitin $1 \mathrm{~g}$ cured 95\% (42 out of 45 men and all of 14 women) with infections caused by penicillinase producing Neisseria gonorrhoeae (PPNG) strains and $98 \%$ (all of 38 men and 13 out of 14 women) with nonPPNG infections, giving an overall cure rate of $96 \%$. The rate of postgonococcal urethritis (PGU) in men treated with $1 \mathrm{~g}$ cefoxitin was $28 \%$. No serious side effects of treatment were observed in patients treated with either dose. The failure rate of $7 \%$ in men infected with PPNG strains who were treated with the $1 \mathrm{~g}$ dose is, however, disturbing. We therefore recommend that intramuscular cefoxitin $2 \mathrm{~g}$ and oral probenecid $1 \mathrm{~g}$ may be used to treat uncomplicated gonorrhoea, especially in areas where PPNG strains are common.

\section{Introduction}

For several years the gonococcus has shown itself to be a highly adaptable organism that resisted the onslaught of antibiotics. Relative resistance to penicillins emerged in the 1950s, when strains with minimum inhibitory concentrations (MICs) of more than $0.018 \mathrm{mg} / 1$ penicillin appeared. By the early 1970 s, MICs of penicillin were $0.5 \mathrm{mg} / 1$ or more for $45 \%$ or more of gonococcal strains isolated in South East Asia and the Philippines. ${ }^{1}$ To overcome the problem of increasing resistance, the dose of procaine penicillin $G$ increased from 1.2 MIU to 4.8 MIU combined with probenecid.

Even such high dose penicillin treatment, however, was outmoded by the emergence of penicillinase producing strains of Neisseria gonorrhoeae (PPNG) in 1976. ${ }^{2}$ PPNG strains have now become endemic in South East Asia, accounting for 35-60\% of gonococcal infections, ${ }^{3}$ and threaten to spread throughout the

Address for reprints: Dr K B Lim, Middle Road Hospital, 250 Middle Road, Singapore 0718, Republic of Singapore

Accepted for publication 20 December 1985 world. The Centers for Disease Control (CDC) of the United States of America recommends the use of spectinomycin $2 \mathrm{~g}$ by intramuscular injection to treat patients (and their sexual partners) who have confirmed infection with PPNG strains or who are likely to have acquired gonorrhoea in areas of high prevalence of PPNG strains. ${ }^{4}$

Spectinomycin is costly, however, and its use has been complicated by the emergence of spectinomycin resistant PPNG strains in England (Easmon CSF, et al , unpublished observation) and South Korea. ${ }^{5}$ The CDC made provision for cases of spectinomycin resistance by recommending cefoxitin $2 \mathrm{~g}$ intramuscularly and probenecid $1 \mathrm{~g}$ by mouth. ${ }^{4}$ In 1979 Berg et al reported that this regimen cured all of 54 men, including 21 infected with PPNG ${ }^{6}$ In evaluating lower doses of cefoxitin, Sanchez et al (unpublished observation) found that $1 \mathrm{~g}$ of cefoxitin with probenecid cured all of 20 men with gonorrhoea (12 infected with PPNG and eight with non-PPNG strains). The same workers, however, found $500 \mathrm{mg}$ of cefoxitin to be totally ineffective. As the number of patients assessed was small, we repeated the study evaluating the efficacy of cefoxitin $1 \mathrm{~g}$ plus probenecid. Before doing so we ran a pilot study to 
confirm that cefoxitin $2 \mathrm{~g}$ and probenecid was still effective. The methodology, results of treatment, and antibiotic susceptibility test results are reported.

\section{Patients and methods}

This study was conducted in the outpatient department of Middle Road Hospital, Singapore, during August 1984 to September 1985.

\section{STUDY POPULATION}

Men whose urethral smears and women whose cervical smears showed Gram negative intracellular diplococci were selected for the study. Patients with a history of allergy to penicillin, evidence of complicated gonorrhoea or coexisting syphilis, and those who had received antibiotic treatment within the preceding two weeks were excluded. Also excluded were patients aged under 18, prostitutes, those whose cultures for $N$ gonorrhoeae were negative, and those who had sexual intercourse before the first follow up. Verbal consent was obtained from all patients.

\section{ISOLATION OF $N$ GONORRHOEAE AND TESTS OF SUSCEPTIBILITY TO CEFOXITIN}

Urethral and endocervical specimens obtained with sterile platinum loops and cotton tipped sterile swabs, respectively, were immediately inoculated on to modified Thayer-Martin medium, incubated at $36^{\circ} \mathrm{C}$ in an atmosphere of carbon dioxide, and examined after 24 to 48 hours. Gonococcal isolates were identified by colonial morphology, oxidase reaction, and Gram stained microscopic appearance. Penicillinase production was tested for by the paper acidometric method of Sng et al. ${ }^{7}$ Confirmed isolates were tested for susceptibility to cefoxitin by the agar dilution method described previously. ${ }^{8}$ Cefoxitin was used in doubling dilutions from $0 \cdot 125 \mathrm{mg} /$ to $8 \mathrm{mg} / \mathrm{l}$ for measuring MICs.

TREATMENT AND FOLLOW UP

We treated 25 patients with cefoxitin $2 \mathrm{~g}$ and probenecid $1 \mathrm{~g}$ and 137 with cefoxitin $1 \mathrm{~g}$ plus probenecid $1 \mathrm{~g}$. We obtained urethral smears and cultures from men, and cervical cultures only from women three to seven days and 10 to 20 days after treatment. Cure was based on negative test results three to seven days after treatment. Patients treated with cefoxitin $1 \mathrm{~g}$ who attended at least one follow up visit were evaluated for side effects of treatment. Men on the same regimen who attended for follow up on days 10 to 20 were evaluated for postgonococcal urethritis (PGU), which was diagnosed by the presentce of five or more polymorphonuclear neutrophils per $1000 \times$ microscopic field, no Gram negative diplococci, and a negative culture for $N$ gonorrhoeae. The Venereal Diseases Research Laboratory (VDRL) slide test was performed before treatment and six weeks and three months afterwards.

\section{Results}

\section{STUDY POPULATION}

We selected 162 patients for treatment, 26 of whom were excluded from the study because four had negative cultures before treatment; four had been reinfected, and 18 defaulted after treatment. Thus there remained 136 patients aged 18 to 60 for evaluation of treatment response. We treated 25 (four men and 21 women) with cefoxitin $2 \mathrm{~g}$ and probenecid $1 \mathrm{~g}$ and 111 ( 83 men and 28 women) with cefoxitin $1 \mathrm{~g}$ and probenecid $1 \mathrm{~g}$. We evaluated 109 patients for side effects of treatment and assessed 67 men for PGU.

\section{ERADICATION OF N GONORRHOEAE}

Table I shows the details of response to treatment. Cefoxitin in a $2 \mathrm{~g}$ dose cured all 25 patients treated, including 11 with infections due to PPNG strains. Cefoxitin in a $1 \mathrm{~g}$ dose cured $56(95 \%)$ of patients infected with PPNG strains and 51 (98\%) of 52 patients infected with non-PPNG strains. The overall cure rate was $96 \%(107 / 111)$. In the men $42(93 \%)$ of 45 cases due to PPNG strains and all of 38 cases due to non-PPNG strains were cured, giving an overall cure rate of $96 \%(80 / 83)$. In the women all of 14 cases

TABLE I. Response to treatment of 136 patients with gonorrhoea

\begin{tabular}{lllll}
\hline & \multicolumn{4}{l}{ No cured/No treated (\% cured): } \\
\cline { 2 - 5 } Treatment & Strains & Men & Women & Total \\
\hline Cefoxitin $2 \mathrm{~g}+$ probenecid $1 \mathrm{~g}$ & PPNG & $3 / 3(100)$ & $8 / 8(100)$ & $11 / 11(100)$ \\
& Non-PPNG & $1 / 1(100)$ & $13 / 13(100)$ & $14 / 14(100)$ \\
Cefoxitin $1 \mathrm{~g}+$ probenecid $1 \mathrm{~g}$ & PPNG & $42 / 45(93)$ & $14 / 14(100)$ & $56 / 59(95)$ \\
& Non-PPNG & $38 / 38(100)$ & $13 / 14(93)$ & $51 / 52(98)$ \\
\hline
\end{tabular}

PPNG = penicillinase ( $\beta$ lactamase) producing Neisseria gonorrhoeae. 
due to PPNG strains and 13 (93\%) of 14 cases due to non-PPNG strains were cured, giving an overall cure rate of $(96 \%)(27 / 28)$.

\section{SENSITIVITY TO ANTIBIOTIC}

Table II shows the results of antibiotic susceptibility tests. The geometric mean MICs were $1.31 \mathrm{mg} /$ for PPNG strains and $1.21 \mathrm{mg} /$ for non PPNG strains. MICs of $2 \mathrm{mg} / 1$ or more were found in $55 \%$ of the PPNG isolates and $51 \%$ of the non-PPNG isolates. The difference in the distribution of the MICs of PPNG and non-PPNG strains using the Wilcoxon rank sum test was not significant $(p>0.05)$.

TABLE II. Susceptibility of 70 strains of Neisseria gonorrhoeae to cefoxitin

\begin{tabular}{|c|c|c|c|c|c|c|}
\hline \multirow[b]{2}{*}{ Strains } & \multicolumn{6}{|c|}{ No with MICs of $\mathrm{mg} / \mathrm{l}$} \\
\hline & 0.25 & 0.5 & 1 & 2 & 4 & $\begin{array}{l}\text { Geometric mean } \\
\text { MICs }(m g /)\end{array}$ \\
\hline $\begin{array}{l}\text { PPNG }(n=33) \\
\text { Non-PPNG } \\
(n=37)\end{array}$ & $\begin{array}{l}2 \\
4\end{array}$ & $\begin{array}{l}7 \\
5\end{array}$ & $\begin{array}{l}6 \\
9\end{array}$ & $\begin{array}{l}12 \\
15\end{array}$ & $\begin{array}{l}6 \\
4\end{array}$ & $\begin{array}{l}1 \cdot 31 \\
1 \cdot 21\end{array}$ \\
\hline
\end{tabular}

PPNG=penicillinase $(\boldsymbol{\beta}$ lactamase $)$ producing Neisseria gonorrhoeae.

POSTGONOCOCCAL URETHRITIS (PGU)

PGU was diagnosed in 19 (28\%) of the 67 men assessed.

\section{SIDE EFFECTS}

Fifteen (14\%) of 109 patients assessed complained spontaneously of side effects. Of these, 13 complained of pain at the injection site, one complained of pruritus at the injection site, and one complained of transient giddiness. All symptoms resolved within 24 hours without medication.

\section{Discussion}

Cefoxitin is a cephamycin derivative with good in vitro activity against $\boldsymbol{N}$ gonorrhoeae. Its stability to penicillinase and its pharmacokinetic characteristics showed that it had potential for treating gonorrhoea caused by PPNG and non-PPNG strains.$^{910}$ Initial studies by Berg et al in 1979 showed cefoxitin $2 \mathrm{~g}$ and probenecid $1 \mathrm{~g}$ to be effective, ${ }^{6}$ and a $1 \mathrm{~g}$ dose of cefoxitin has been shown to be equally effective in a small number of patients (Sanchez PL, et al, unpublished observation). Because of the ability of the gonococcus to develop resistance, we ran a pilot study to assess whether cefoxitin $2 \mathrm{~g}$ with probenecid was still effective in the mid-1980s. Our results suggest that it is, and all 25 patients treated, including 11 infected with PPNG strains, were cured. In the main study evaluating the efficacy of cefoxitin $1 \mathrm{~g}$ with probenecid, $95 \%(56)$ of 59 patients (45 men and 14 women) infected with PPNG strains and $98 \%(51)$ of 52 patients (38 men and 14 women) infected with non-PPNG strains were cured, giving an overall cure rate of $96 \%(107 / 111)$. It was not possible to gauge accurately the efficacy of cefoxitin $1 \mathrm{~g}$ in women because of the small number of women assessed in this study. In men, only $93 \%$ (42) of 45 infected with PPNG strains were cured in contrast to $100 \%$ of 38 infected with non-PPNG strains.

Antibiotic susceptibility tests showed that $55 \%$ of PPNG strains and $51 \%$ of non-PPNG strains isolated in this study had MICs of cefoxitin of $2 \mathrm{mg} / \mathrm{l}$ or more. The geometric mean MICs for these strains were 1.31 and $1.21 \mathrm{mg} / \mathrm{l}$ respectively. These MICs were substantially higher than those obtained by Berg et al in efficacy studies conducted in the Phillipines in $1979 .{ }^{6}$ Their sensitivity tests showed geometric mean MICs of $0.46 \mathrm{mg} / 1$ for PPNG strains and $0.60 \mathrm{mg} / \mathrm{l}$ for nonPPNG strains. The distribution of the MICs for our strains was similar to that reported by Brown et al, who found that $54.1 \%$ of PPNG and $66.4 \%$ of non-PPNG isolates in Thailand in $1981 \mathrm{had}$ MICs of $2 \mathrm{mg} / \mathrm{l}$ or more. ${ }^{11}$ These results may indicate that South East Asian strains of $N$ gonorrhoeae are less susceptible to cefoxitin.

Cefoxitin 1 or $2 \mathrm{~g}$ with probenecid is a safe regimen, which has no major side effects. Pain from intramuscular cefoxitin of variable severity was experienced by all patients when they were asked specifically. The severity of pain may be reduced by incorporating lignocaine in the injection, as is recommended by the manufacturers. Cefoxitin $1 \mathrm{~g}$ is ineffective in treating PGU and additional treatment is required for this. Cefoxitin $2 \mathrm{~g}$, rather than $1 \mathrm{~g}$, should be used with probenecid in areas where PPNG strains are prevalent, as the $1 \mathrm{~g}$ dose may be associated with a 7\% failure rate in men infected with PPNG strains.

We thank Merck, Sharp and Dohme, USA for supplying cefoxitin, P H Ang and Joanne Foo of Summit Drug (Singapore) for helping to arrange this study, and staff nurses Y L Koh, Y C Tan, and B Y Wong for their help in conducting this study.

\section{References}

1. Sparling PF. Antibiotic resistance in the gonococcus. In: Roberts RB, ed. The gonococcus. New York: Wiley, 1978:111.

2. Phillips I. $\beta$-lactamase producing, penicillin-resistant gonococcus. Lancet 1976,ii:656-7.

3. Centers for Disease Control. Global distribution of penicillinaseproducing Neisseria gonorrhoeae (PPNG). MMWR 
4. United States Department of Health, Education, and Welfare. CDC recommended treatment schedules. Sex Transm Dis 1979;6:38-40.

5. Centers for Disease Control. Spectinomycin-resistant Neisseria gonorrhoeae worldwide. MMWR 1982;31:636-8.

6. Berg SW, Kilpatrick ME, Harrison WO, McCutchan JA Cefoxitin as a single-dose treatment for urethritis caused by penicillinase-producing Neisseria gonorrhoeae. $N$ Engl J Med 1979;301:509-11.

7. Sng EH, Yeo KL, Rajan VS. Simple method for detecting penicillinase-producing Neisseria gonorrhoeae and Staphylococcus aureus. British Journal of Venereal Diseases 1981:57:141-2.
8. Sng EH, Rajan VS, Pang R, Yeo KL. Susceptibility of Neisseria gonorrhoeae to cefotaxime and ceftizoxime. British Journal of Venereal Diseases 1981;57:162-4.

9. Schrogie JJ, Rogers JD, Yeh KC. Pharmacokinetics and comparative pharmacology of cefoxitin and cephalosporins. Rev Infect Dis 1979;1:90-7.

10. Elwell LP, Roberts M, Mayer LW. Plasmid mediated betalactamase production in Neisseria gonorrhoeae. Antimicrob Agents Chemother 1977;11:528-33.

12. Brown $\mathrm{S}$, Warnnissorn $\mathrm{T}$, Biddle J, Pannikabutra $\mathrm{K}$, Traisupa A. Antimicrobial resistance of Neisseria gonorrhoeae in Bangkok: is single-drug treatment passe. Lancet 1982;ii:1366-8. 